\title{
Dexametasona para profilaxis de náuseas y vómitos postoperatorios: efecto sobre la glicemia en pacientes con diabetes mellitus tipo 2 y en no diabéticos sometidos a cirugía laparoscópica
}

\author{
CLAUDIO E. NAZAR, GHISLAINE C. ECHEVARRÍA,
} HÉCTOR J. LACASSIE, RODRIGO A. FLORES, HERNÁN R. MUÑOZ

\section{Effects on blood glucose of prophylactic dexamethasone for postoperative nausea and vomiting in diabetics and non-diabetics}

Background: Postoperative nausea and vomiting (PONV) prophylaxis with dexamethasone may produce significant hyperglycemia in the postoperative period. Aim: To evaluate if this effect is of greater severity in type 2 diabetics compared with non-diabetic patients. Material and Methods: Forty non-diabetic and thirty type 2 diabetic patients undergoing laparoscopic cholecystectomy were studied in a prospective and double-blind fashion manner. Patients were randomly distributed into 4 groups: Group I, non-diabetics control $(n=20)$, Group II, non-diabetics dexamethasone $(n=20)$, Group III, type 2 diabetics control $(n=15)$, and Group $I V$, type 2 diabetics dexamethasone $(n=15)$. Immediately after induction, patients in groups I and III received isotonic saline and patients in the dexamethasone groups received $8 \mathrm{mg}$ iv of the steroid. Capillary blood glucose concentrations were measured at baseline and every 2 hours during the first 12 hours since the start of surgery. A linear mixed effect model, adjusted for baseline capillary glucose concentration, age and duration of surgery was used to analyze the data. Results: No effect of the presence of diabetes mellitus was observed in the evolution of glucose concentrations. There was a difference in capillary glucose concentrations between patients who received dexamethasone and placebo that started 2 hours post-intervention, reaching a mean maximum difference of $34 \mathrm{mg} / \mathrm{dl}$ (adjusted model, $p<0.001$ ) at 10 hours post-intervention. Conclusions: In this study, Type 2 diabetic patients did not show a higher susceptibility than non-diabetics to develop postoperative hyperglycemia after the use of prophylactic dexamethasone for PONV.

(Rev Med Chile 2011; 139: 755-761).

Key words: Dexamethasone; Diabetes mellitus, type 2; Postoperative nausea and vomiting.

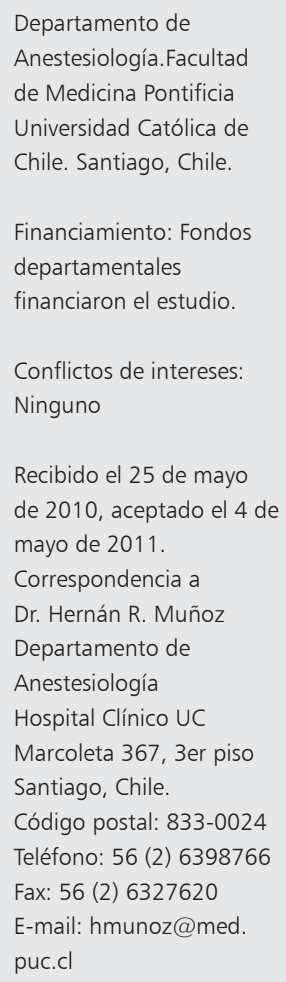

I

as náuseas y vómitos postoperatorios (NVPO) son un problema habitual en los pacientes quirúrgicos. Sin tratamiento, ocurre en 20 a 30\% de la población quirúrgica y en hasta 70 a $80 \%$ en los pacientes de alto riesgo ${ }^{1}$. Los efectos adversos de las NVPO van desde molestias para el paciente hasta potencial morbilidad y en el caso de los pacientes ambulatorios es causa de rehospitalizaciones con incrementos significativos de los costos de atención. 
La dexametasona, sola o administrada en combinación con otras drogas antieméticas, es una droga efectiva para la profilaxis de $\mathrm{NVPO}^{1,2}$. Tiene un buen perfil de costo-efectividad e índice terapéutico, sin embargo, en pacientes sometidos a diferentes procedimientos quirúrgicos se ha descrito hiperglicemia luego de su administración en las dosis habituales usadas para profilaxis de $\mathrm{NVPO}^{3-6}$.

Nuestra hipótesis es que luego de la administración de dexametasona profiláctica para NVPO los pacientes con diabetes mellitus tipo 2 tienen mayor riesgo de desarrollar hiperglicemia en el período postoperatorio comparados con pacientes no diabéticos. El objetivo de este estudio es comparar el efecto de la dexametasona profiláctica sobre las concentraciones de glucosa sanguínea postoperatoria en pacientes diabéticos tipo 2 con el de no diabéticos sometidos a colecistectomía laparoscópica.

\section{Material y Método}

Luego de obtener la aprobación del Comité de Ética de la Escuela de Medicina de la Pontificia Universidad Católica de Chile, Santiago, Chile y consentimiento informado escrito de los pacientes, se estudiaron pacientes no diabéticos y diabéticos tipo 2 sometidos a colecistectomía laparoscópica electiva. Se incluyeron pacientes mayores de 18 años, con un índice de masa corporal (IMC) entre 20 y $30 \mathrm{~kg} \mathrm{~m}^{-2}$ y la presencia o no, según el grupo, de diabetes mellitus tipo 2 de acuerdo a los criterios diagnósticos del año 2006 de la $\mathrm{WHO}^{7}$. Se excluyeron pacientes que estuviesen recibiendo corticoides, insulina, drogas vasoactivas y aquellos que hubiesen recibido glucosa o hipoglicemiantes orales el día de la cirugía. Todos los pacientes tenían un ayuno preoperatorio de al menos 6 horas y no hubo administración de fluidos endovenosos (iv) previo a la inducción anestésica.

La anestesia se indujo con propofol 2-3 mg $\mathrm{kg}^{-1} \mathrm{y}$ se administró rocuronio $0,6 \mathrm{mg} \mathrm{kg}^{-1}$ para facilitar la intubación traqueal. La anestesia se mantuvo con isoflurano o sevoflurano (0,5-1,0 $\mathrm{MAC})$ en óxido nitroso y oxígeno $\left(\mathrm{FiO}_{2} 0,5\right)$ y fentanyl titulados para mantener estabilidad hemodinámica (presión arterial media y frecuencia cardiaca $\pm 20 \%$ de la basal). La ventilación se con- troló y ajustó para mantener $\mathrm{ETCO}_{2}$ entre 30-35 $\mathrm{mmHg}$. Los fluidos intraoperatorios incluyeron solución Ringer lactato y no hubo administración de soluciones que contuvieran glucosa. Durante el período postoperatorio, el ayuno se mantuvo por 12 horas luego de terminada la cirugía y se dejó a todos los pacientes con una solución glucosada al $5 \%$ con electrolitos a $80 \mathrm{ml} / \mathrm{h}$ iv. La técnica quirúrgica fue estandarizada y realizada por el mismo equipo quirúrgico.

Previo a la inducción anestésica, los pacientes diabéticos y no diabéticos fueron distribuidos aleatoriamente mediante números generados computacionalmente en cuatro grupos: Grupo I, no diabéticos control $(\mathrm{n}=20)$, Grupo II, no diabéticos dexametasona $(\mathrm{n}=20)$, Grupo III, diabéticos tipo 2 control $(\mathrm{n}=15)$ y Grupo IV, diabéticos tipo 2 dexametasona $(\mathrm{n}=15)$. Inmediatamente después de la inducción (T0), los pacientes de los 2 grupos control recibieron solución salina $(\mathrm{NaCl} 0,9 \%) 2$ $\mathrm{ml}$ iv y los pacientes de los grupos de la dexametasona, recibieron $8 \mathrm{mg}$ iv (equivalentes a $2 \mathrm{ml}$ ) de esta droga. Las jeringas con la droga en estudio y el placebo fueron etiquetadas con el número de asignación aleatoria del paciente.

Los niveles de glucosa sanguínea fueron medidos por la técnica de "hemoglucotest" de una muestra de sangre capilar obtenida de un pulpejo del paciente con un lancetero y usando el monitor de glicemia Accucheck Sensor (Roche, Mannheim, Alemania) calibrado diariamente. Se obtuvo una medición basal antes de la inducción anestésica y luego cada 2 horas desde T0 hasta completar 12 horas. Todas las mediciones fueron hechas por investigadores que desconocían el grupo al cual pertenecían los pacientes. Todos los pacientes recibieron $60 \mathrm{mg}$ de ketorolaco iv luego de la inducción anestésica y $4 \mathrm{mg}$ de ondansetrón iv al concluir el procedimiento quirúrgico. El manejo del dolor postoperatorio fue realizado con una infusión iv de ketorolaco 90 mg en 24 horas más $3 \mathrm{mg}$ de morfina iv si el paciente tenía un puntaje EVA $>3($ EVA $=$ escala visual análoga con puntaje de $0=\sin$ dolor, a $10=$ mayor dolor imaginable). Los eventos eméticos postoperatorios fueron tratados con $1 \mathrm{mg}$ de droperidol iv seguido de una segunda dosis de $4 \mathrm{mg}$ de ondansetrón iv si fuese necesario. Se registró el uso de drogas no anestésicas en el período de estudio. No se realizaron intervenciones para manejar la glicemia durante todo el estudio. 


\section{Estadística}

Usando un valor $\alpha=0,05$ y $\beta=0,10$, se necesi$\tan 15$ pacientes por grupo para que una diferencia de $45 \mathrm{mg} \mathrm{dl}^{-1}$ en los valores máximos de glucosa al comparar dos grupos sea estadísticamente significativa. Se eligió el valor de $45 \mathrm{mg} \mathrm{dl}^{-1}$, porque estudios previos ${ }^{5,8}$ mostraron que con esta diferencia, los pacientes que recibían dexametasona alcanzaban valores superiores a $180 \mathrm{mg} \mathrm{dl}^{-1}$, cifra que es considerada el límite superior aceptable en pacientes no críticos ${ }^{9}$. Decidimos comenzar con un tamaño muestral de 20 pacientes por grupo ante posibles pérdidas de pacientes o datos incompletos; sin embargo, comenzado el estudio, nos dimos cuenta de la dificultad de encontrar pacientes diabéticos tipo 2 con IMC $<30 \mathrm{~kg} \mathrm{~m}^{-2}$ que se sometieran a una colecistectomía laparoscópica. En consecuencia, se redujo los grupos III y IV a 15 pacientes (mínimo permitido). Se evaluó normalidad de los datos utilizando la prueba de Shapiro-Wilk y se realizó una transformación logarítmica a los valores de la glucosa capilar previo al análisis, dado que no presentaban una distribución normal. Posteriormente, estos valores fueron analizados utilizando modelos lineales mixtos, dada la necesidad de modelar correctamente las matrices de varianzas/covarianzas (var/cov) de los datos ${ }^{10}$. Puesto que nuestro objetivo era comparar la evolución de los niveles de glucosa en el tiempo con respecto a los distintos niveles del factor diabetes y factor droga, se creó un modelo que incluyó, no sólo los factores principales Diabetes (presencia/ausencia), Droga (solución fisiológica/ dexametasona) y Tiempo (medidas repetidas), sino también sus interacciones y la Glucosa capilar basal como covariable (modelo 1). Ya que la edad promedio de los grupos con y sin diabetes y la duración de la cirugía diferían estadísticamente, se creó un segundo modelo (modelo 2) incluyendo estas covariables. Usando la prueba de razón de verosimilitud ambos modelos fueron comparados (igual matriz de var/cov), obteniéndose un mejor ajuste con el modelo 2 (modelo final, $\mathrm{p}=0,011)^{11}$.

Los datos demográficos e intraoperatorios continuos fueron analizados utilizando la prueba t de Student o análisis de varianza (ANOVA) seguido de la prueba de Tukey HSD, según correspondiera. Las proporciones fueron comparadas utilizando la prueba de chi-cuadrado de Pearson sobre la base de 2.000 réplicas, cuando la frecuencia esperada fue menor a $5^{12}$.

Se consideró significativo un valor de $\mathrm{p}<$ 0,05 . Los resultados se expresan como promedios \pm desviación estándar (DE). Todos los análisis fueron realizados utilizando SAS versión 9.2 (SAS Institute, Cary, NC).

\section{Resultados}

Los datos generales se muestran en la Tabla 1. Todos los pacientes fueron catalogados como ASA I-II y no recibieron premedicación. La edad promedio de los pacientes diabéticos (53 años, IC95\%: 47-59) fue mayor que en los no diabéticos (40 años, IC95\%: 39-47), ( $p=0,006$ con $\mathrm{t}$ de Student). Se obtuvo un total de 490 mediciones de glucosa capilar, sin perder ninguna medición. Se observó una diferencia significativa en la duración

Tabla 1. Características de los pacientes y datos intraoperatorios

\begin{tabular}{|c|c|c|c|c|}
\hline & $\begin{array}{l}\text { Grupo I } \\
(n=20)\end{array}$ & $\begin{array}{l}\text { Grupo II } \\
(n=20)\end{array}$ & $\begin{array}{l}\text { Grupo III } \\
(n=15)\end{array}$ & $\begin{array}{r}\text { Grupo IV } \\
(n=15)\end{array}$ \\
\hline Edad (años) & $45 \pm 15$ & $41 \pm 12^{*}$ & $56 \pm 14^{*}$ & $51 \pm 19$ \\
\hline Peso (kg) & $69 \pm 10$ & $64 \pm 9$ & $67 \pm 12$ & $73 \pm 9$ \\
\hline Talla (cm) & $164 \pm 7$ & $164 \pm 6$ & $162 \pm 8$ & $167 \pm 8$ \\
\hline Género (masculino/femenino) & $6 / 14$ & $3 / 17$ & $4 / 11$ & $8 / 7$ \\
\hline IMC $\left(\mathrm{kg} \mathrm{m}^{-2}\right)$ & $25,4 \pm 2,8$ & $23,9 \pm 2,8$ & $25,4 \pm 3,3$ & $26,0 \pm 2,7$ \\
\hline Duración de la cirugía (min) & $51 \pm 11^{*+}$ & $59 \pm 17$ & $66 \pm 16^{*}$ & $66 \pm 20^{+}$ \\
\hline Tiempo de anestesia (min) & $74 \pm 14$ & $82 \pm 18$ & $88 \pm 15$ & $87 \pm 24$ \\
\hline Consumo de fentanyl $\left(\mu \mathrm{g} \mathrm{kg}^{-1}\right)$ & $4,8 \pm 0,8$ & $5,1 \pm 0,9$ & $5,5 \pm 1,4$ & $4,8 \pm 1,1$ \\
\hline
\end{tabular}

Valores son promedios $\pm \mathrm{DE}$, excepto género (números absolutos). ${ }^{*} \mathrm{y}^{\dagger}=\mathrm{p}<0,05$. 


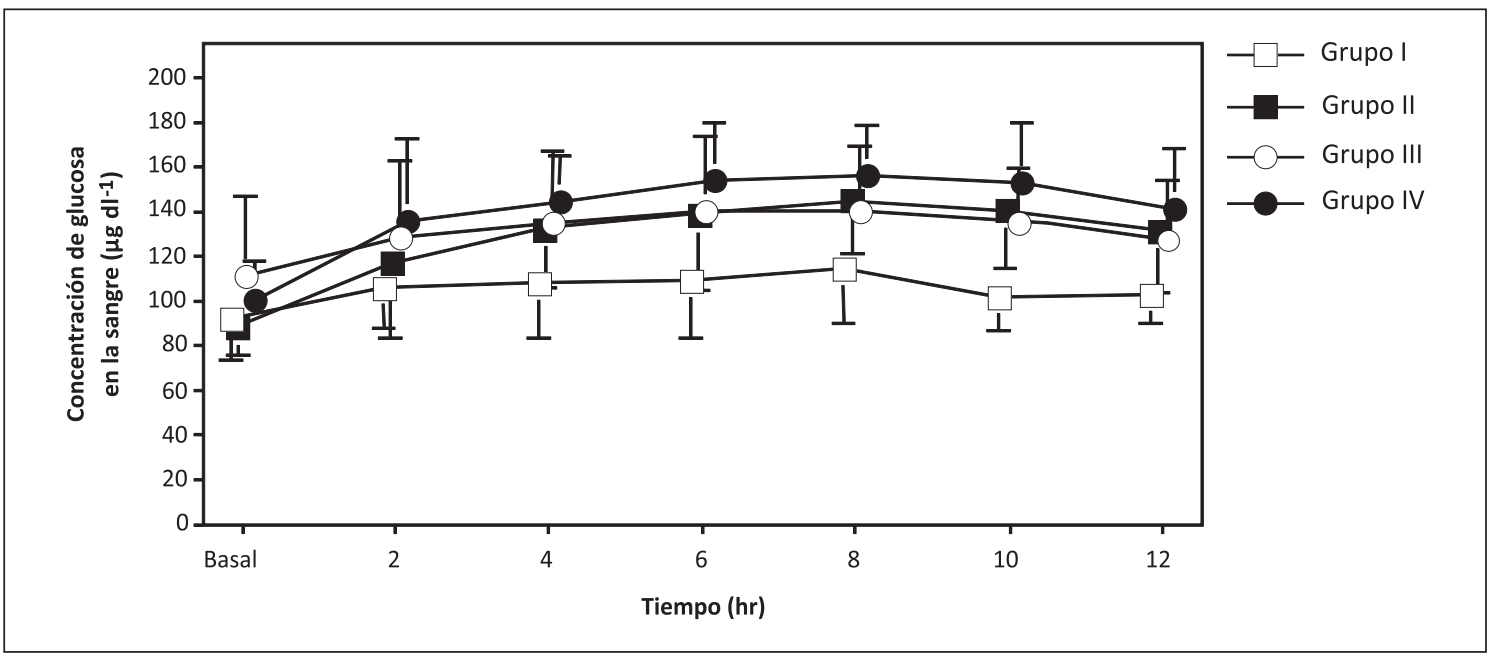

Figura 1. Muestra los valores de glucosa capilar basales y cada 2 horas en los 4 grupos luego de la administración de dexametasona o solución salina (promedio $\pm \mathrm{DE}$ ). Sólo la interacción entre Droga (solución salina/dexametasona) y Tiempo fue estadísticamente significativa $(p=0,044)$. La presencia o no de diabetes no fue un factor significativo relacionado con el cambio de la concentración de glucosa capilar (modelo ajustado por valor basal, edad y tiempo quirúrgico).

de la cirugía entre los grupos $(\mathrm{p}=0,016$, Tabla 1$)$. El promedio de la glucosa capilar basal del grupo diabéticos fue de $105 \mathrm{mg} \mathrm{dl}^{-1}$ [IC95\%: 95-115] y de $90 \mathrm{mg} \mathrm{dl}^{-1}$ [IC95\%: 85-94] para el grupo no diabéticos ( $\mathrm{p}=0,005$ con $\mathrm{t}$ de Student).

Todos los pacientes presentaron un aumento en el valor de la glucosa capilar, pero este aumento fue más pronunciado en aquellos pacientes que recibieron dexametasona (interacción entre Droga (solución fisiológica/dexametasona) y Tiempo fue estadísticamente significativa $(\mathrm{p}=0,044)$ ) (Figura 1). El análisis post hoc de la interacción (comparaciones de efecto simple) reveló que la diferencia en la evolución de la glucosa capilar en los grupos con y sin intervención, independiente de la presencia o no de diabetes tipo 2, comenzó a ser significativa a las 2 horas post-operatorias (diferencia promedio de $14 \mathrm{mg} \mathrm{dl}^{-1}$ ajustada por valor de glucosa basal, edad y tiempo quirúrgico, $\mathrm{p}=0,021$ ), alcanzando la máxima diferencia promedio a las 10 horas post intervención $\left(34 \mathrm{mg} \mathrm{dl}^{-1}, \mathrm{p}<0,001\right)$, diferencia que se mantuvo hasta el fin del estudio $\left(27 \mathrm{mg} \mathrm{dl}^{-1}\right.$, $\mathrm{p}<0,001)$. El resto de las interacciones no fueron estadísticamente significativas.

El porcentaje de pacientes con glicemia $\geq 180$ $\mathrm{mg} \mathrm{dl}{ }^{-1}$, en cualquier momento luego de T0 fue de $5 \%, 20 \%, 13 \%$ y $27 \%$ en los grupos I, II, III y IV, respectivamente $(\mathrm{p}=0,983)$. Al final del estudio, el porcentaje de pacientes con glicemias $\geq 180 \mathrm{mg}$ $\mathrm{dl}^{-1}$ fue $0 \%$ en el grupo I, $10 \%$ en el grupo II, 7\% en el grupo III y $7 \%$ en el grupo IV $(p=0,994)$ y ningún paciente presentó valores de glicemia sobre $200 \mathrm{mg} \mathrm{dl}^{-1}$ (Figura 2).

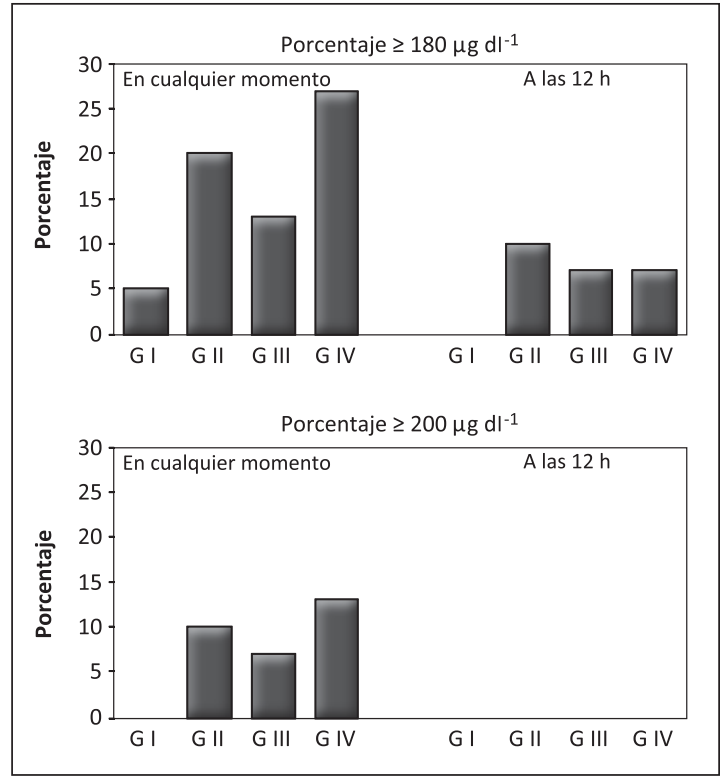

Figura 2. Muestra el porcentaje de pacientes en los 4 grupos que presentaron concentraciones de glucosa sanguínea mayores a $180 \mathrm{mg} \mathrm{dl}^{-1}$ (Panel superior) o $200 \mathrm{mg} \mathrm{dl}^{-1}$ (Panel inferior) en cualquier momento del estudio y 12 horas después de la administración de dexametasona o solución salina. 


\section{Discusión}

El principal hallazgo de este estudio es que, contrario a nuestra hipótesis, los pacientes con diabetes mellitus tipo 2 sometidos a colecistectomía laparoscópica no parecen ser más susceptibles que los pacientes no diabéticos a desarrollar hiperglicemia luego de la administración profiláctica de $8 \mathrm{mg}$ de dexametasona iv para NVPO.

La hiperglicemia es un efecto adverso frecuente de los corticoides y está mediada por la estimulación de la neoglucogénesis hepática, desarrollo de insulinorresistencia e inhibición de la captura de glucosa en los tejidos periféricos ${ }^{13}$. La hiperglicemia asociada al uso de dexametasona ha sido demostrada en diferentes grupos de pacientes quirúrgicos ${ }^{3,5,6,8}$. Sin embargo, aún no ha sido claramente definido el efecto que esta droga pudiese tener en la glicemia de pacientes diabéticos. Hans y cols ${ }^{4}$ encontraron que $10 \mathrm{mg}$ de dexametasona resultaban en glicemias postoperatorias superiores en pacientes diabéticos tipo 2 comparados con no diabéticos. Desafortunadamente, este estudio sólo midió valores de glicemia hasta 6 horas después de finalizada la cirugía y es sabido que los valores de glicemia llegan a su máximo después de este período. Además, la falta de un grupo control sin dexametasona, impide cualquier conclusión en cuanto a si los diabéticos pudiesen tener una respuesta más exacerbada a esta droga comparados con no diabéticos. En este estudio, nosotros decidimos extender las mediciones hasta 12 horas después de la administración de la dexametasona para así detectar los valores máximos de glicemia que ocurren entre las 8 a 10 horas después de su administración. Además, incluimos pacientes no diabéticos para evaluar si los diabéticos tienen una respuesta hiperglicémica más pronunciada a la dexametasona que los pacientes "normales". Como se ha demostrado previamente ${ }^{3,8}$, encontramos que los pacientes no diabéticos que recibieron dexametasona al comienzo de la cirugía desarrollan hiperglicemia significativa que alcanza el máximo 8 horas después de la administración y que luego empieza a decaer. Los diabéticos tipo 2 también muestran esta tendencia temporal en los niveles de glicemia, pero, inesperadamente, no mostraron un mayor incremento de la glicemia que los pacientes no diabéticos. Por lo tanto, los valores máximos de glicemia en diabéticos que recibieron y no recibieron dexametasona y en no diabéticos que recibieron dexametasona fueron similares. Estos resultados sugieren que los pacientes diabéticos tipo 2 son igualmente susceptibles a los efectos hiperglicémicos de la dexametasona que los no diabéticos. Si bien no tenemos una explicación clara para este fenómeno, se ha visto que los valores máximos de glicemia luego de la administración de dexametasona están relacionados en forma lineal a los valores de la hemoglobina glicosilada [HbA (1c) $]^{4} \mathrm{o}$, en otras palabras, a la calidad del control de la glicemia. Desafortunadamente, no medimos HbA (1c) como para saber si el potencial buen control de la enfermedad en nuestros pacientes es un factor asociado a la falta de diferencia en la respuesta glicémica en los diabéticos.

Otros autores han postulado que los valores postoperatorios de glicemia en diabéticos a los cuales se les administró dexametasona pudieran ser más difíciles de manejar ${ }^{14}$. Sin embargo, el retorno espontáneo a valores más bajos al final del período de estudio, no apoya esta posibilidad. Estos resultados apoyan el uso, cuando esté indicado, de dexametasona para profilaxis de NVPO en estos pacientes.

Una de las falencias del estudio, que también ha ocurrido en estudios previos ${ }^{4}$, es que los pacientes diabéticos son mayores que los pacientes no diabéticos. Si bien un estudio con no diabéticos de la misma edad hubiera corregido este error, creemos que este hecho no invalida nuestros resultados. De hecho, la comparación de diabéticos que recibieron y que no recibieron dexametasona muestra una evolución similar en las glicemias, apoyando la conclusión de que los diabéticos no son particularmente más susceptibles a desarrollar hiperglicemia por dexametasona. Si bien puede ser argumentado que usamos una dosis de dexametasona mayor a la recomendada en algunas guías de manejo de $\mathrm{NVPO}^{2}$, la probabilidad que dosis menores aumenten el riesgo de hiperglicemia, como para invalidar nuestros resultados, es baja. Una crítica a este estudio es la falta de tratamiento de la hiperglicemia en pacientes diabéticos. Sin embargo, debido a que ya se había demostrado el efecto autolimitado de la dexametasona, decidimos dejar el período de estudio libre de tratamiento ya que los pacientes fueron monitorizados cada 2 horas para medir glicemias y eventuales síntomas. De hecho, el porcentaje de pacientes diabéticos, ya sea con o sin dexametasona, con valores de glicemia sobre $180 \mathrm{mg} \mathrm{dl}^{-1}$, un valor considerado 
como límite superior de la glicemia esperada en unidades de pacientes no críticos $^{8}$, fue similar al de los no diabéticos que recibieron dexametasona. Además, doce horas después de la administración de la dexametasona, todos los pacientes tuvieron glicemias menores a $200 \mathrm{mg} \mathrm{dl}^{-1}$, es decir, dentro del rango actualmente aceptado en el período postoperatorio de pacientes diabéticos ${ }^{14}$.

Finalmente, una limitación importante del estudio tiene relación con la metodología de medición de la glicemia. El uso de sangre capilar en aparatos portátiles, como el utilizado en este estudio, pueden presentar diferencias significativas en los resultados de la medición cuando se comparan con las obtenidas en el laboratorio de referencia ${ }^{15}$.

Diversas características de los pacientes, como la presencia de hipotensión, anemia, mala perfusión periférica, hipotermia y uso de drogas vasoactivas, entre otros, aumentan la posibilidad de lecturas erróneas con los aparatos portátiles ${ }^{15,16}$.

Los errores de medición se reducen significativamente en los pacientes hospitalizados fuera de una unidad de cuidados intensivos $y$, al igual que en estos últimos, habitualmente son sobreestimaciones de los valores obtenidos en el laboratorio de referencia con el consiguiente riesgo de no detectar hipoglicemias ${ }^{17}$. Los pacientes de nuestro estudio, por estar en buenas condiciones, se fueron a su pieza en el postoperatorio y ninguno de ellos presentó alguna de las condiciones anteriores que reconocidamente aumentan la imprecisión de los aparatos portátiles. Además, el hecho que los aparatos portátiles tiendan a sobreestimar los valores de glicemia también apoya el hecho que los diabéticos tipo 2 no parecen ser particularmente susceptibles a la dexametasona en las dosis utilizadas.

En resumen, contrario a nuestra hipótesis, los pacientes diabéticos tipo 2 no parecen ser más susceptibles que los no diabéticos a desarrollar hiperglicemia luego de profilaxis para NVPO con dexametasona. Por lo tanto, esta droga es una alternativa válida para el manejo de este efecto adverso en este subgrupo de pacientes.

\section{Referencias}

1. Gan TJ, Meyer TA, Apfel CC, Chung F, Davis PJ, Habib AS et al. Society for Ambulatory Anesthesia guidelines for the management of postoperative nausea and vomiting. Anesth Analg 2007; 105: 1615-28.
2. Apfel CC, Korttila K, Abdalla M, Kerger H, Turan A, Vedder I et al. A factorial trial of six interventions for the prevention of postoperative nausea and vomiting. N Engl J Med 2004; 350: 2441-51.

3. Pasternak JJ, McGregor DG, and Lanier WL. Effect of single-dose dexamethasone on blood glucose concentration in patients undergoing craniotomy. J Neurosurg Anesthesiol 2004; 16: 122-5.

4. Hans P, Vanthuyne A, Dewandre PY, Brichant JF, Bonhomme V. Blood glucose concentration profile after $10 \mathrm{mg}$ dexamethasone in non-diabetic and type 2 diabetic patients undergoing abdominal surgery. Br J Anaesth 2006; 97: 164-70.

5. Nazar CE, Lacassié HJ, López RA, Muñoz HR. Dexamethasone for postoperative nausea and vomiting prophylaxis: effect on glycaemia in obese patients with impaired glucose tolerance. Eur J Anaesthesiol 2009; 26: 318-21.

6. Eberhart LH, Graf J, Morin AM, Stief T, Kalder M, Lattermann R, et al. Randomised controlled trial of the effect of oral premedication with dexamethasone on hyperglycaemic response to abdominal hysterectomy. Eur J Anaesthesiol 2011; 28: 195-201.

7. World Health Organization: Definition and Diagnosis of Diabetes Mellitus and Intermediate Hyperglycemia. Report of a WHO/IDF Consultation. 2006: Geneva, Switzerland.

8. Lukins MB, Manninen PH. Hyperglycemia in patients administered dexamethasone for craniotomy. Anesth Analg 2005; 100: 1129-33.

9. Moghissi ES, Korytkowski MT, DiNardo M, Einhorn D, Hellman R, Hirsch IB, et al. American Association of Clinical Endocrinologists and American Diabetes Association consensus statement on inpatient glycemic control. Endocr Pract 2009; 15: 353-69.

10. Littell RC, Pendergast J, Natarajan R. Modelling covariance structure in the analysis of repeated measures data. Stat Med 2000; 19: 1793-819.

11. Davis CS. Statistical methods for the analysis of repeated measurements. Springer texts in statistics. New York: Springer, 2002.

12. Higgins JJ. An introduction to modern nonparametric statistics. Pacific Grove, CA: Brooks/Cole, 2004.

13. Schimmer BP, Parker KL. Adrenocorticotropic Hormone; Adrenocortical Steroids and Their Synthetic Analogs; Inhibitors of the Synthesis and Actions of Adrenocortical Hormones. En: L.S. Goodman, A. Gilman, L.L. Brunton, J.S. Lazo, and K.L. Parker, Goodman $\&$ Gilman's the pharmacological basis of therapeutics. New York: McGraw-Hill; 2006. p. 1587-612.

14. Lipshutz AK, Gropper MA. Perioperative glycemic con- 
trol: an evidence-based review. Anesthesiology 2009; 110: 408-21.

15. Dungan K, Chapman J, Braithwaite SS, Buse J. Glucose measurement: confounding issues in setting targets for inpatient management. Diabetes Care 2007; 30: 403-9.
16. Fahy BG, Coursin DB. Critical glucose control: the devil is in the details. Mayo Clin Proc 2008; 83: 394-7.

17. Hoedemaekers CW, Klein Gunnewiek JM, Prinsen MA, Willems JL, Van der Hoeven JG. Accuracy of bedside glucose measurement from three glucometers in critically ill patients. Crit Care Med 2008; 36: 3062-6. 